\title{
Homology to peptide pattern for annotation of carbohydrate-active enzymes and prediction of function
}

\author{
P. K. Busk* (D, B. Pilgaard, M. J. Lezyk, A. S. Meyer and L. Lange
}

\begin{abstract}
Background: Carbohydrate-active enzymes are found in all organisms and participate in key biological processes. These enzymes are classified in 274 families in the CAZy database but the sequence diversity within each family makes it a major task to identify new family members and to provide basis for prediction of enzyme function. A fast and reliable method for de novo annotation of genes encoding carbohydrate-active enzymes is to identify conserved peptides in the curated enzyme families followed by matching of the conserved peptides to the sequence of interest as demonstrated for the glycosyl hydrolase and the lytic polysaccharide monooxygenase families. This approach not only assigns the enzymes to families but also provides functional prediction of the enzymes with high accuracy.

Results: We identified conserved peptides for all enzyme families in the CAZy database with Peptide Pattern Recognition. The conserved peptides were matched to protein sequence for de novo annotation and functional prediction of carbohydrate-active enzymes with the Hotpep method. Annotation of protein sequences from 12 bacterial and 16 fungal genomes to families with Hotpep had an accuracy of 0.84 (measured as F1-score) compared to semiautomatic annotation by the CAZy database whereas the dbCAN HMM-based method had an accuracy of 0.77 with optimized parameters. Furthermore, Hotpep provided a functional prediction with $86 \%$ accuracy for the annotated genes. Hotpep is available as a stand-alone application for MS Windows.
\end{abstract}

Conclusions: Hotpep is a state-of-the-art method for automatic annotation and functional prediction of carbohydrate-active enzymes.

Keywords: Carbohydrate-active enzymes, Genomics, Annotation, Software

\section{Background}

Carbohydrate-active enzymes are produced by all organisms to accomplish enzymatic modification of carbohydrate-containing compound both intra- and extracellularly. Hence, this enzyme group is relevant for understanding central biological processes such as sugar metabolism, protein glycosylation and, on an ecological level, for global biomass synthesis and degradation. It is not surprising that carbohydrate-active enzymes are used in medical and industrial biotechnology. The CAZy database (http://www.cazy.org/) was founded in 1991 and contains a unique classification of carbohydrate-active enzymes including carefully curated information about enzyme

\footnotetext{
* Correspondence: pbus@kt.dtu.dk

Department of Chemical and Biochemical Engineering, Technical University of Denmark, Søltofts Plads, Building 229, 2800 Kgs. Lyngby, Denmark
}

sequence, structure and function [1]. Currently, the publicly available information in the CAZy database consists of almost 400.000 unique protein sequences classified in more than 300 families.

Despite the abundant information in the CAZy database, de novo annotation of carbohydrate-active enzymes is not a trivial task. State-of-the-art methods involve automatic identification by matching the sequences of interest to protein models generated directly from sequences in the CAZy database or indirectly from protein domain models from other databases or by BLAST search followed by manual curation of the data [1-4].

Entirely automatic annotation methods have been developed based on hidden Markov model (HMM) recognition of all or a subset of the enzymes in the CAZy database and are available as web-based services [5-7]. E.g., the dbCAN method was made by refining 
HMM models from the Conserved Domain Database to fit the families in the CAZy database and supplementing the database with new HMM models for the families in the CAZy database that are not modelled in the Conserved Domain Database [7].

Even when it is possible to annotate a protein to a specific family this does not necessarily allow an exact prediction of its enzymatic activity. This is due to that the classification of the carbohydrate-active enzymes in the CAZy database is based on protein sequence and structure similarity [1]. Thus, in many cases the classification does not reflect enzymatic activity [1]. Hence, proteins with identical enzymatic activity are classified in different families and most of the families contain proteins with different enzymatic activities.

Identification of short, conserved motifs can be used to group related protein sequences and will often pinpoint proteins with the same enzymatic activity $[8,9]$. Furthermore, the method Homology to Peptide Pattern (Hotpep) matches the short, conserved motifs to undescribed protein sequences to obtain a fast, sensitive and precise annotation of carbohydrate-active enzymes to families [10]. Moreover, when experimental data on enzymatic activity is available Hotpep allows prediction of the enzymatic activity of the proteins. In practice, the experimental data on enzyme activity collected in the CAZy database can be used to predict the enzymatic activity of approximately $75 \%$ of the carbohydrate-active enzymes in a genome with $80 \%$ accuracy $[9,10]$.

We used the method Peptide Pattern Recognition (PPR) to identify short, conserved sequence motifs for all enzyme families in the CAZy database. The peptide patterns were combined with Hotpep to obtain a standalone software for automatic annotation and functional prediction of carbohydrate-active enzymes. As an example, to illustrate the workability of the approach, annotation of protein sequences from 12 bacterial and 16 fungal genomes was addressed. Hotpep had an F1 score of 0.86 (sensitivity $=0.88$, precision $=0.84$ ) for predicting carbohydrate-active enzymes in 12 bacterial genomes and an F1 score of 0.82 (sensitivity $=0.77$, precision $=0.88$ ) for predicting carbohydrate-active enzymes in 16 fungal genomes compared to semiautomatic annotation by the CAZy database tools for carbohydrate-active enzyme annotation [1, 4]. Moreover, Hotpep correctly predicted the activity of $86 \%$ of the characterized carbohydrateactive enzymes in the CAZy database.

The carbohydrate binding modules $(\mathrm{CBM})$ are not defined as carbohydrate-active enzymes per se but are carbohydrate binding domains within multidomain carbohydrate-active enzymes [11]. Using short, conserved peptides for the CBM families in the CAZy database Hotpep annotates the CBMs with an F1 score of 0.87 .
The Hotpep stand-alone application is available for download from Sourceforge for use on desktop computers with the MS Windows operative system.

\section{Implementation}

Development and testing of Hotpep for carbohydrateactive enzymes followed a number of steps as outlined (Fig. 1).

\section{Protein sequences}

The first step was to download sequences for all members of each carbohydrate-active enzyme family in the CAZy database (www.cazy.org [1]) from Genbank (https://www.ncbi.nlm.nih.gov/ [12]) in August, 2016. The CBM families were downloaded in February, 2017. Sequences that were $100 \%$ redundant or $100 \%$ identical to a part of another sequence were removed.

\section{Identification of short, conserved peptides}

PPR was used for identification of short, conserved peptides in each family of carbohydrate-active enzymes as previously described $[9,10,13]$. Briefly, for each family

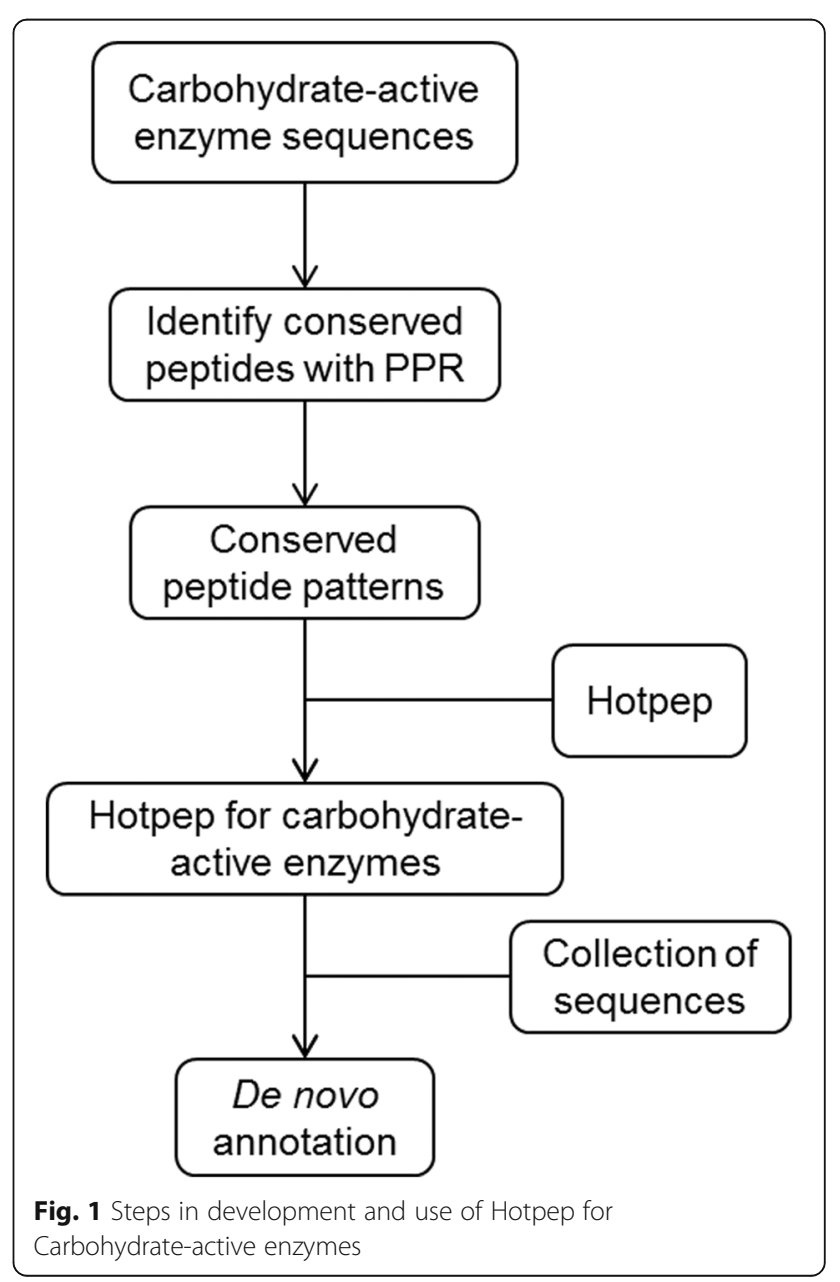


PPR found the largest group of proteins that contained at least 10 of 70 conserved hexamer peptides. The length of the conserved peptides (hexamers), the number of conserved peptides per protein (10) and the total number of conserved peptides per group (70) were chosen as they were the conditions that gave the best rate of prediction of protein function in empirical testing of peptide lengths from trimers to decamers, $5-40$ conserved peptides per protein and $30-200$ conserved peptides per group [9]. Moreover, the minimum frequency of each conserved peptide in a group was 0.20 as this threshold gives the best rate of prediction of protein function [9]. For CBM domains the parameters 30 conserved hexapeptides per PPR group and 3 conserved peptides per protein were used for PPR analysis.

The first group of proteins identified by this method was named group 1. Next, PPR found the second largest group of proteins, not including any proteins from group 1. This group of proteins was named group 2 and so on. The analysis was stopped when less than five proteins were grouped together.

In this way a number of groups consisting of a list of protein sequences and a list of conserved peptides were generated for each family in the CAZy database. Groups including proteins with a described enzyme activity as reported in the CAZy database were assigned the same function as the enzymes as previously described [9].

For AA families 9, 10 and 11 the conserved peptide lists of the previously described expanded families were used [13].

\section{Sequence collections}

Genome-annotated protein products ("*;protein.faa.gz" files) were downloaded from Genbank for 12 bacterial (Table 1) and 16 fungal species (Table 2). For comparison of annotation from genomes and from predicted proteins the files "-genomic.fna.gz (genome assembly) and *_protein.faa.gz (protein products annotated on the genome assembly) for the following fungi Thermothelomyces thermophile (Accession: GCF_000226095.1), Talaromyces stipitatus (Accession: GCA_000003125.1), Botryobasidium botryosum (Accession: GCA_000697 705.1), Coprinopsis cinerea (Accession: GCA_0001828 95.1), Serendipita indica (Accession: GCA_000313 545.1), Mucor circinelloides (Accession: GCA_000401 635.1) and Rhizopus delemar (Accession: GCA_00014 9305.1) were downloaded from Genbank.

\section{Annotation with Hotpep}

Genomic fragments were annotated as previously described [10]. Annotation of protein products from genome assemblies was performed on full-length predicted protein sequences essentially as described [9]. Briefly, protein sequence was given a score for each groupspecific peptide lists for each family by:

1. Finding all the conserved peptides from the list that were present in the sequence.

2. Sum the frequency of these peptides to obtain the group-specific frequency score.

A hit was considered significant if the protein sequence:

1. Included three or more conserved peptides from a group.

2. The frequency score for the peptides was higher than 1.0

3. The conserved peptides represented at least ten amino acids of the protein sequence.

If a protein satisfied all three conditions it was assigned to the family and to the PPR group with the

Table 1 Bacterial strains and accession numbers

\begin{tabular}{llll}
\hline Name & Phylum & Isolated from & Accession numbers \\
\hline $\begin{array}{l}\text { Bacteroides cellulosilyticus WH2 } \\
\text { Caldicellulosiruptor saccharolyticus DSM8903 }\end{array}$ & Bacteroidetes & Gut and stomach & GCA_000463315.1 \\
& Firmicutes & Wood & Thermophilic anaerobe \\
Deinococcus peraridilitoris DSM19664 & Deinococcus-Thermus & Coastal desert & GCA_000317835.1 \\
Desulfotomaculum gibsoniae DSM7213 & Firmicutes & Freshwater ditch & GCA_000233715.3 \\
Enterobacter lignolyticus SCF1 & Proteobacteria & Tropical forest soil & GCA_000164865.1 \\
Melioribacter roseus P3M-2 & Ignavibacteriae & Wooden surface of a chute & GCA_000279145.1 \\
Prevotella ruminicola 23 & Bacteroidetes & Gut & GCA_000025925.1 \\
Rhodococcus jostii RHA1 & Actinobacteria & Hexachlorocyclohexane-contaminated soil & GCA_000014565.1 \\
Ruminiclostridium thermocellum ATCC27405 & Firmicutes & Soil/manure & GCA_000015865.1 \\
Teredinibacter turnerae T7901 & Proteobacteria & Intracellular in shipworm & GCA_000023025.1 \\
Thermacetogenium phaeum DSM12270 & Firmicutes & thermophilic anaerobic methanogenic reactor & GCA_000305935.1 \\
Thermoanaerobacterium thermosaccharolyticum DSM571 & Firmicutes & Soil & GCA_000145615.1 \\
\hline
\end{tabular}


Table 2 Fungal strains (basidiomycotae) and accession numbers

\begin{tabular}{llll}
\hline Name & Order & Life style & Accession numbers \\
\hline Postia placenta & Polyporales & Brown rot & GCA_000006255.1 \\
Fomitopsis pinicola & Polyporales & Brown rot & GCA_000344655.2 \\
Gloeophyllum trabeum & Gloeophyllales & Brown rot & GCA_000344685.1 \\
Coniophora puteana & Boletales & Brown rot & GCA_000271625.1 \\
Dacryopinax sp. & Dacrymycetales & Brown rot & GCA_000292625.1 \\
Tremella mesenterica & Tremellales & Mycoparasite & GCA_000271645.1 \\
Dichomitus squalens & Polyporales & White rot & GCA_000275845.1 \\
Trametes versicolor & Polyporales & White rot & GCA_000271585.1 \\
Fomitiporia mediterranea & Hymenochaetales & White rot & GCA_000271605.1 \\
Auricularia delicata & Auriculariales & White rot & GCA_000265015.1 \\
Punctularia strigosozonata & Corticiales & White rot & GCA_000264995.1 \\
Heterobasidion annosum & Russulales & White rot & GCA_000320585.2 \\
Stereum hirsutum & Russulales & White rot & GCA_000264905.1 \\
Phanerochaete_carnosa & Polyporales & White rot & GCA_000300595.1 \\
Ceriporiopsis subvermispora & Polyporales & White rot & GCA_000320605.2 \\
Phlebiopsis gigantea & Polyporales & White rot & GCA_000832265.1 \\
\hline
\end{tabular}

highest group-specific frequency score. Moreover, if this group had been assigned a function by the PPR analysis, the same function was predicted for the protein [9].

Hotpep including the conserved peptide patterns described here is available for download as an application for the MS Office operative system from Sourceforge.

\section{Annotation with dbCAN}

The protein products from each genome were annotated de novo with the dbCAN web service for protein annotation with standard parameters and with optimized parameters (E-value $<10^{-18}$; coverage $>0.35$ for bacteria and $\mathrm{E}$-value $<10^{-17}$; coverage $>0.45$ for fungi) by downloading scripts and HMMs as described (http:// csbl.bmb.uga.edu/dbCAN/annotate.php, [7]).

\section{Statistical analysis}

The following values were calculated for pairwise comparison of two annotation methods:

True positives $=$ Number of hits found by both screening methods. False positives $=$ Number of proteins found by the screening method being tested but not by the reference method. False negatives $=$ Number of proteins found by the reference method but not by the screening method being tested.

Sensitivity was calculated as True positives/(True positives + False negatives); Precision (positive prediction value) was calculated as True positives/(True positives + False positives) and F1 score (the harmonic mean of precision and sensitivity) was calculated as $(2 \times$ True positives $) /(2 \times$ True positives + False positives + False negatives).

\section{Results and discussion}

Short, conserved peptides identified in the carbohydrateactive enzyme from the glycoside hydrolase families in the CAZy database can be used for fast, efficient and reliable approach for annotation by the Hotpep method [10]. Moreover, groups of carbohydrate-active proteins sharing the same short, conserved peptides do often have the same enzymatic activity [9]. Thus, by comparing the rich information on experimentally characterized enzymes in the CAZy database with the PPR grouping of the enzymes it is possible to predict the enzymatic activity of the uncharacterized members of the groups with $80 \%$ accuracy. In this way, a functional prediction was obtained for $72 \%$ of the annotated glycoside hydrolases in 39 fungal genomes [10].

To accomplish automatic annotation of all carbohydrateactive enzymes with Hotpep we downloaded all sequences in the families of the five enzyme classes: Carbohydrate esterases (CE), Glycoside hydrolases (GH), Auxiliary activities (AA), Polysaccharide lyases (PL) and Glycosyl transferases (GT). A total of 594,121 accession numbers were found in the CAZy database and reduced to 380,269 non-redundant protein sequences before each family was sorted into groups of proteins sharing up to 70 short, conserved hexapeptides and assignment of function to each group containing more than two functionally characterized members (Additional file 1). In total 36\% of the 5590 PPR groups for all enzyme families included functionally characterized proteins. These groups with associated functions contained $65 \%$ of the PPR-grouped proteins. For the glycoside hydrolases, $41 \%$ of the groups included functionally characterized proteins and a total of $74 \%$ of all proteins, in agreement 
with the previous report of a functional prediction of $72 \%$ of the glycoside hydrolases [10].

For the CBM class of carbohydrate-binding modules we found 71,253 accession numbers in the CAZy database resulting in 45,048 non-redundant protein sequences. Due to the short length of most CBM domains $[7,11]$ it was uncertain whether the standard parameters of 70 conserved peptides per PPR group and 10 conserved peptides per protein were optimal for annotation of CBMs. Therefore, different parameters for PPR were tested for classification of the isolated CBM domains followed by Hotpep annotation of the full-length proteins and comparison to the annotation in the CAZy database. There was little variation in the $\mathrm{F} 1$ score $(0.83-0.87)$ within the range of tested parameters (Additional file 2) in agreement with the notion that PPR groups are fairly stable within a large range of parameters [9]. The parameters 30 conserved peptides per PPR group and 3 conserved peptides per protein gave the highest $\mathrm{F} 1$ score of 0.87 and were chosen for annotation of CBMs.

Hotpep annotates proteins by matching the lists of conserved peptides of a group to the protein sequences of interest $[10,13,14]$. Any sequence that fulfills a number of criteria (see Implementation) of which the most important is that the sequence should include at least three of the conserved peptides, will be annotated to the protein group. We combined Hotpep with the lists of conserved peptides for all enzyme families in the CAZy database to an application that can identify members of all carbohydrate-active enzyme families and CBMs. The AA9, AA10 and AA11 conserved peptides were substituted with the AA9exp, AA10exp and AA11exp conserved peptides that represent a more complete description of the sequence variation in these families [13]. The complete lists of peptides and frequencies are available for download at Sourceforge together with the accession numbers of the sequences for each group and the library of EC functional scores for each group.

The input for annotation with Hotpep is a text file with predicted protein sequences in fasta format. The algorithm is started by double-clicking the Hotpep icon. This will open a DOS prompt, where the user writes the name of the input file containing the fasta-formatted protein sequences (Fig. 2).

Hotpep screens the input sequences for members of all families in the CAZy database. This will take 5 $20 \mathrm{~min}$ for all predicted genes in a bacterial or fungal genome. Several genomes can be annotated in parallel by running Hotpep several times. The results files are saved in six directories, one for each class of carbohydrate-active enzymes, one for the CBMs and two summary files: One with the number of hits for each family and one with the accession number of each hit and the families annotated for this hit (Fig. 3a). The latter file gives an overview of the number and families for multidomain enzymes.

The results for each enzyme class is a number of text files (Fig. 3b) prepared for import into MS Excel, LibreOffice or similar spreadsheet applications (Fig. 4). The columns in the spread sheet designates the group where the sequence is annotated, the name of the sequence, the sum of the frequencies of the conserved peptides $[10,14]$, the number of conserved peptides, the protein sequence, length of the sequence and the sequences of the conserved peptides. In addition, the directories contain a subdirectory with files including prediction of the activities of the enzymes arranged according to EC class (Fig. 3c). As the CBMs are binding modules associated to enzyme domains the predicted function is often the predicted function of the associated enzyme domain as described in the CAZy database. The files with functional prediction contain a column with the prediction of the enzymatic function according to EC class (Fig. 5). The information in this column consists of one or more EC numbers each followed by a colon and a number designating the sum of the number of conserved peptides in each characterized protein in the group. The higher this number, the more proteins in the group have the enzymatic activity represented by the EC number. E.g.; in family GH43 group 71 there are 48 conserved peptide matches to enzymes characterized as endoxylanases (EC 3.2.1.8) (Fig. 5). For family GH8 group 3 there are 65 conserved peptide matches to enzymes characterized as endoxylanases (EC 3.2.1.8) but also 41 conserved peptide matches to enzymes characterized as exo-oligoxylanase (EC 3.2.1.156) in addition to matches to enzymes with other activities (Fig. 5). Hence, expression and enzymatic characterization of the sequence with the accession number WP_029428720.1 annotated to this group is necessary to decide whether it is an endoxylanase or an exo-oligoxylanase as the scores for these two activities are similar.

This method correctly predicts $80-95 \%$ of enzyme activities $[9,10]$. To test this further, we used Hotpep to predict the function of 8812 experimentally characterized carbohydrate-active enzymes (Additional file 3). Hotpep correctly predicted the function of $86 \%$ of the enzymes. This result supports the previous finding that proteins sharing conserved peptides often but not always have the same activity [9]. Hence, enzymatic activities for individual sequences predicted by Hotpep should be used as a guideline for functional characterization. In an analysis of annotation of glycosyl hydrolases from ORFs in genome fragments with Hotpep it was found that the glycosyl hydrolases that were overlooked by Hotpep could be detected when the full-length amino acid sequence of the enzymes were used for annotation [10]. This finding suggests that more true positive hits are 


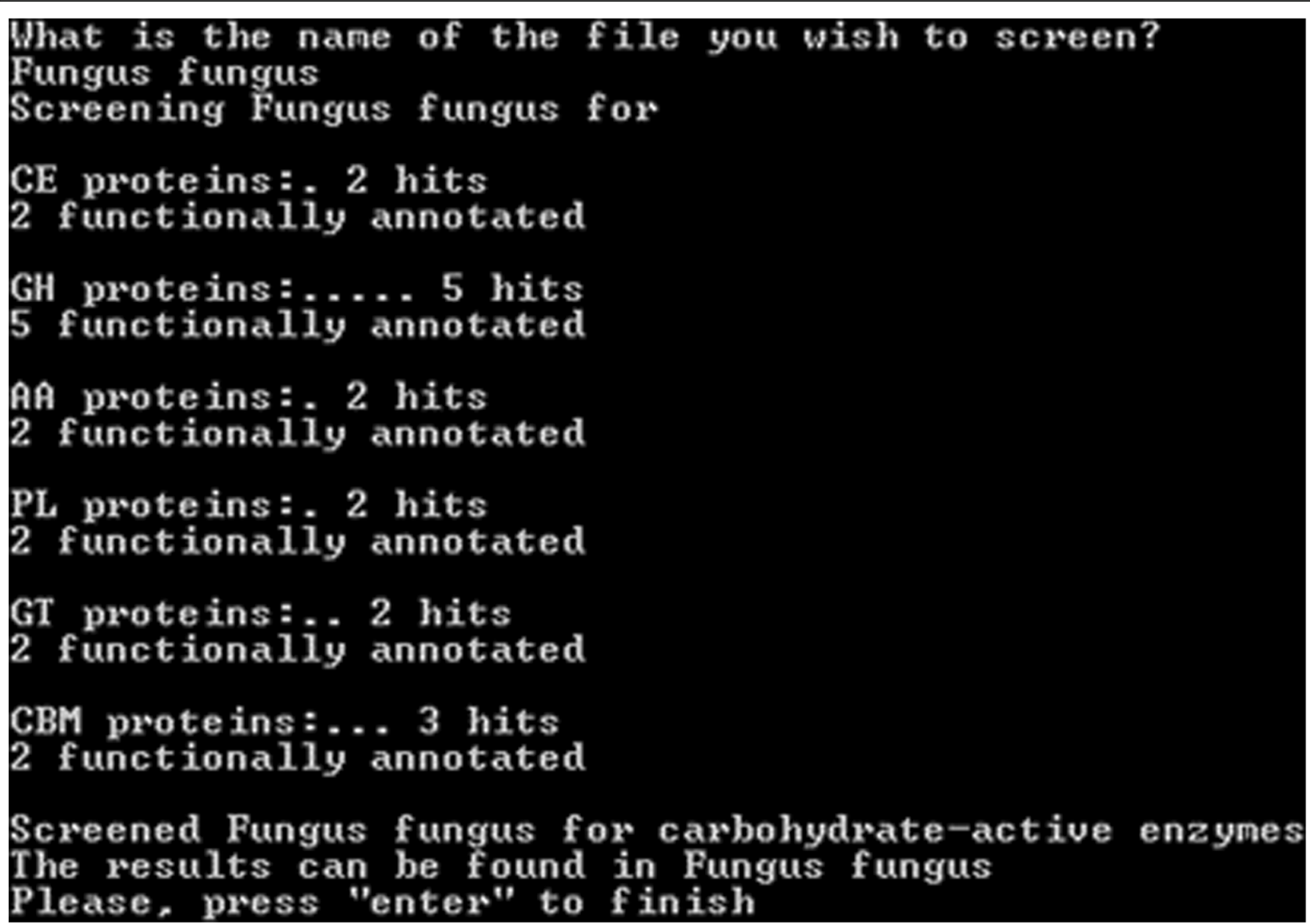

Fig. 2 Hotpep user interface. Double-clicking on the Hotpep icon opens a DOS promt where the name of the sequence directory (e.g., "Fungus fungus") is entered

obtained by examining full-length coding regions rather than ORFs containing single exons. To test this notion we compared the annotation of all carbohydrate-active enzymes in seven fungal genomes to annotation of predicted proteins from the same genomes. The fungi were selected to include genome assemblies and predicted proteins from different research groups to avoid methodical bias. The results showed that $31 \%$ more carbohydrate-active enzymes were found by annotation of the predicted proteins from the genomes compared to annotation of ORFs in fragments of the genomes (Additional file 4) in agreement with the previous report [10].

\section{a}

Name

D. CBM

d. CE

d. $\mathrm{GH}$

d. GT

d. $\mathrm{PL}$

d. $A A$

b. orfs1

2. summary_all_enzymes

bummary_all_families b

$$
\text { Type }
$$

File folder

File folder

File folder

File folder

File folder

File folder

Text Document

Text Document

Text Document

Name Type

Fig. 3 Organization of the Hotpep output. a. The output is delivered in the sequence directory with one directory for each enzyme class in the CAZy database, a file containing a summary of the results and a file with all the families found for each accession number. $\mathbf{b}$. Each of the class directories contains files with the hits for each family, a summary and a directory with functional predictions. c. The folder with functional predictions contains files for each EC number found and a summary 


\begin{tabular}{|c|c|c|c|c|c|c|}
\hline GH3 & seq_name & Frequency & hits & sequence & length & peptides \\
\hline 1 & >WP_029426049.1 glya & 11,3 & 16 & MKKVFRKTSLLLATALMGVTGMQAQKAPQDMDRFIDTLMKK & 773 & PFGYGL,RDPRWG,DVLFGD,FG \\
\hline 1 & $>$ WP_029426038.1 glya & 11,3 & 16 & KKILKRTSLULISALMSIAAAQAQKSPQDMDRFIDALMKKMT & 784 & D,FGYGLS,RDPRWG, \\
\hline 1 & $>$ WP_029428699.1 bete & 6,65 & 9 & KTLKIFSACLFLLPFVSCTQVANKGSDAATEKKVESLLSKMTL & 750 & PFGYGL,RDPRWG,GEDTYL,FGI \\
\hline 1 & $>$ WP_029426308.1 betê & 5,33 & 7 & KDLVLKGLRITVVSLALSGCNTPDNLYLDPAQPIEARVDNLM & 769 & DPRWGR,FGYGLS,GYGLSY,SRL \\
\hline 2 & $>$ WP_029426618.1 glya & 4,76 & 15 & IMSFYRLDEKYKSVILKINIMKSGMKCITTGLFKIFILTLSWGSLC & 793 & NFEYYS, VVSDWG, VLLKNE, FEY \\
\hline 4 & $>$ WP_029426465.1 glya & 9,32 & 28 & MKRKLQLLTGIGCLCLCFLSCSQPPYKNPALSPEERANDLVGRL' & 863 & EALHGV,TVFPQA,INIFRD,PRU \\
\hline 4 & >WP_029427148.1 glya & 8,32 & 24 & MKRWILIGMVVASGMTIQAQNKLPEKFPYQDTSLTAEERADC & 864 & FRDPRW,GRGQET,FGHGLS,RD \\
\hline 4 & >WP_033160524.1 hype & 7,11 & 21 & MINKKKIIIVFTLLLGGALMKTFAQSFKYPFQNPKLDVEERVKD & 881 & NIFRDP,RWGRGQ,ETYGED,IFF \\
\hline 4 & $>$ WP_029427455.1 glya & 9 & 20 & MKKRWIILWLSAMTLNVTAQNEPYKNPELSPSERAWDLLKRN & 864 & FPQAIG,TYGEDP,GHGLSY,HGL \\
\hline 4 & $>$ WP_029429014.1 bete & 4,38 & 15 & KKIIATMAIGACLCSCGGSQKEVYKDSTAPVKDRVEDLLKRN & 766 & ,TYGEDP, KFRL \\
\hline 10 & \$429017.1 betâ & 10, & 27 & 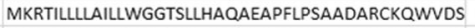 & 1000 & PGHGL \\
\hline 10 & 9429016.1 betê & 10 & 26 & KLFLFLULFSCCARFDAQTHRLPAPQSPVTPVE & 1046 & TT,GLGFDK,DGE \\
\hline & & & & & & \\
\hline
\end{tabular}

Fig. 4 Hotpep output. An output files with hits for the GH3 family opened in MS Excel. The columns (from left to right) contain the group where the sequence is annotated, the name of the sequence, the sum of the frequency of the conserved peptides, the number of conserved peptides, the protein sequence, length of the sequence and the sequences of the conserved peptides

Hence, although exon-intron structure of eukaryotic genes makes them difficult to predict [15] a higher sensitivity in prediction of carbohydrate-active enzymes is obtained by annotating from predicted proteins rather than from ORFs in genome fragments.

Annotation with Hotpep of predicted proteins from 12 bacterial genomes was compared to state-of-the-art semiautomatic annotation reported in the CAZy database [1]. The selected genomes were from bacteria with different lifestyles including bacteria known to degrade extracellular carbohydrates.

The CAZy database reported slightly less carbohydrateactive enzymes than Hotpep for the 12 bacterial genomes (Table 3). We have previously found that Hotpep annotation of fungal genomes are largely in agreement with the results reported in the CAZy database and that the differences between the annotations may be due to genes overlooked by either Hotpep or in the CAZy database [10]. This is a natural effect of the fact that the families in the CAZy database are growing as new members are discovered and some of the families are redefined [1]. E.g.; the lytic polysaccharide monooxygenases (LPMOs) originally classified in the GH61 and CBM33 families $[9,16,17]$ were later reclassified to the AA9, AA10 and AA11 families $[18,19]$. In view of this plasticity of the CAZy database it is difficult to precisely determine the correct annotation of carbohydrate-active enzymes in a given dataset [7]. However, if the annotation reported in the CAZy database is defined as correct, then it means that the Hotpep annotation has a sensitivity of 0.88 and a precision of 0.84 (Table 3). This gives an F1 score of 0.86 , which means that the methods on average agree on $86 \%$ of the number of predicted carbohydrate-active enzymes.

It was reported that automatic identification with the HMM signatures in dbCAN is a highly precise and sensitive method for annotation of carbohydrate-active enzymes [7]. Annotation of the 12 bacterial genomes with the dbCAN web service (http://csbl.bmb.uga.edu/ $\mathrm{dbCAN/annotate.php)} \mathrm{gave} \mathrm{a} \mathrm{higher} \mathrm{number} \mathrm{of} \mathrm{hits} \mathrm{than}$ the annotation in the CAZy database resulting in a sensitivity similar to Hotpep but with lower precision and F1 score (Table 3). However, annotation of the 12 bacterial genomes with the downloaded dbCAN HMMs and optimized parameters [7] gave a lower number of hits than the annotation in the CAZy database resulting in slightly higher sensitivity, precision and F1 score than Hotpep (Table 3). Thus, although the downloadable dbCAN is more difficult to use than the web service as the user has to both download the dbCAN HMMs and install the HMMER 3.0 package [7] the extra effort pays

\begin{tabular}{|c|c|c|c|c|c|c|c|c|}
\hline fam & group & Functions & seq_name & sequence & length & peptides & Frequency & hits \\
\hline GH5 & 82 & 3.2.1.8:148,3.2.1.4:2 & $>$ WP_029428723.1 & IMKRIIKVFFFVGFILLSSCSDNTIC & 655 & VNLHGF,TQTYSP,FSET & 44,5 & 67 \\
\hline GH30 & 12 & 3.2.1.8:5 & >WP_029426201.1 & MNLKLPLLLGCCLFAAITACAETS & 491 & DGFGAA,RISIGC,SDFS & 35,2 & 53 \\
\hline GH5 & 82 & 3.2.1.8:148,3.2.1.4:2 & $>$ WP_029426354.1 & : MKNITNVFYEFLIALCCLMSSSAI & 1205 & VVMRPP,MFELAN,DP & 19 & 24 \\
\hline GH43 & 71 & 3.2.1.8:48 & >WP_029426373.1 & IMNKRITLFLITLLTVCGVQSQNN & 894 & TDMVNW,IDDDGQ,A' & 15,4 & 28 \\
\hline GH10 & 21 & 3.2.1.8:158 & >WP_029428724.1 & MKHTKKILGTMLLTAAAVVATSC & 721 & AWDVVN,FYWQDY,V & 11 & 25 \\
\hline GH30 & 12 & 3.2.1.8:5 & >WP_029426189.1 & MKNKRLFTYVFLFGLLVNGSACC & 516 & EYTCCD,TYFVKW, HNY & 9,95 & 16 \\
\hline GH10 & 46 & 3.2.1.8:22 & $>$ WP_029428714.1 & I MKLKYLALSVCAAALMSCNSDK & 897 & RIKGWD,YYNDYG,YPL & 8,81 & 10 \\
\hline GH10 & 46 & 3.2.1.8:22 & >WP_026367873.1 & IMKNKIFLLALLIGFSLYSCGSNST & 371 & LYYNDY,YYNDYG,GAN & 8,64 & 10 \\
\hline GH8 & 16 & 3.2.1.8:70,3.2.1.156:7 & >WP_029426464.1 & |MKNLFYLLLCLIAGASCSQADPT & 419 & PSYHVP,SYHVPA,YHV & 7 & 7 \\
\hline GH10 & 16 & 3.2.1.8:151 & >WP_029429021.1 & IMKKVKSTLSVGKRIILLSLCMTM| & 919 & IVNRYK,DVVYAW,VN & 6,84 & 19 \\
\hline GH10 & 10 & 3.2.1.8:311,3.2.1.55:17,3.2.1.4:11 & $>$ WP_029427770.1 & IMKHLFKFSLCALALTMGANTGF. & 716 & YAWDVV,AWDVVN,V & 6,22 & 11 \\
\hline GH8 & 3 & 3.2.1.8:65,3.2.1.156:41,3.2.1.132:11,3.2.1.x:10,3.2.1.4:7 & >WP_029428720.1 & IMKLTTLFAVSVSLILSGFCSLGVC & 417 & EGMSYG,GMSYGM,DV & 4,83 & 8 \\
\hline GH10 & 21 & 3.2.1.8:158 & $>$ WP_029428711.1 & I MKLNRIILPLMACALTWSSCDDC & 772 & VDGIGT,DGIGTQ,NDY & 4,68 & 8 \\
\hline
\end{tabular}

Fig. 5 Hotpep output for functional prediction. Same as Fig. 4 with the addition of a column labelled "Functions" with information on the putative functions of the annotated sequence 
Table 3 Annotation of 12 bacterial genomes

\begin{tabular}{lllll}
\hline Method & CAZy $^{\mathrm{a}}$ & Hotpep & $\begin{array}{l}\text { dbCAN } \\
\text { web }\end{array}$ & $\begin{array}{l}\text { dbCAN } \\
\text { download }\end{array}$ \\
\hline Annotated proteins & 1768 & 1839 & 2300 & 1749 \\
True positives & - & 1546 & 1701 & 1571 \\
False positives & - & 296 & 599 & 178 \\
False negatives & - & 220 & 67 & 197 \\
Sensitivity & - & 0.88 & 0.87 & 0.89 \\
Precision & - & 0.84 & 0.71 & 0.90 \\
F1 score & - & 0.86 & 0.84 & 0.89 \\
\hline
\end{tabular}

${ }^{a}$ www.cazy.org

of in the form of a more accurate annotation. In summary, the comparison of the annotation methods showed that the CAZy database, Hotpep and downloaded dbCAN were most in agreement whereas the dbCAN web service annotates a higher number of genes as encoding carbohydrate-active enzymes.

To assess the performance of Hotpep for identification of eukaryotic genes, 16 fungal genomes that have been sequenced and annotated by The Joint Genome Institute and the CAZy database tools by Hori et al. [4] were selected for annotation. Testing on these genomes has the benefit that many of the carbohydrate-active enzymes from these fungi are not part of the CAZy database and has thus not been part of the dataset used to make the conserved peptide patterns used by Hotpep.

In case of the fungal genomes, Hori et al. [4] found slightly more carbohydrate-active enzymes than Hotpep (Table 4). However, Hotpep had an F1 score of 0.82 relative to the annotation by Hori et al., whereas annotation with dbCAN web service and downloaded dbCAN with optimized parameters only had F1 scores of 0.68 and 0.72 , respectively (Table 4 ). Hence, for annotation of the fungal genes Hotpep and Hori et al. gave the most similar result whereas the dbCAN web service and the downloaded dbCAN predicted a higher number of carbohydrate-active enzymes. Summarizing the results for prediction of bacterial and fungal genes Hotpep had a combined F1 score of 0.84 , dbCAN web service had an

Table 4 Annotation of 16 fungal genomes

\begin{tabular}{lllll}
\hline Method & JGI/CAZy $^{a}$ & Hotpep & $\begin{array}{l}\text { dbCAN } \\
\text { web }\end{array}$ & $\begin{array}{l}\text { dbCAN } \\
\text { download }\end{array}$ \\
\hline Annotated proteins & 3985 & 3534 & 6238 & 4490 \\
True positives & - & 3084 & 3463 & 3057 \\
False positives & - & 450 & 2775 & 1433 \\
False negatives & - & 901 & 522 & 928 \\
Sensitivity & - & 0.77 & 0.87 & 0.77 \\
Precision & - & 0.88 & 0.56 & 0.68 \\
F1 score & - & 0.82 & 0.68 & 0.72 \\
\hline
\end{tabular}

${ }^{\mathrm{a}}$ Hori et al. [4]
F1 score of 0.75 and downloaded dbCAN with optimized parameters had an F1 score of 0.77 .

The F1 score (0.82) for the comparison of Hotpep with Hori et al. [4] for the 16 fungal genomes is a little lower than the F1 score (0.86) for the annotation of the 12 bacterial genomes. However, the fungal genomes were all from basidiomycetes that are less represented in the CAZy database than carbohydrate-active enzymes from ascomycetes and thus may be more difficult to annotate. To assess this possibility we used previously published data [10] to calculate the F1 score for comparison of annotation of six ascomycete genomes by Hotpep and the CAZy database tools for annotation. The few disagreements between the methods were attributed mainly to differences in gene prediction rather than to differences in annotation [10]. In line with this notion, the F1 score for this dataset of ascomycete genes was $0.92 \mathrm{com}$ pared to only 0.82 for the annotation of basidiomycete genes in the present study. This finding suggests that the publicly available CAZy database may not yet account for the complete sequence variation in the carbohydrateactive enzyme families. E.g., the basidiomycete sequences may be underrepresented. This is in agreement with the ongoing addition of new sequences to the CAZy database [1]. A simple expansion of the LPMO enzyme families in the CAZy database by including previously unannotated, publicly available sequences led to the identification of the AA11 enzymes [9] and was shown to give a better representation of the sequence variation of the families, hereby making it possible to identify $31 \%$ more LPMOs in 39 fungal genomes [13]. The current version of Hotpep for annotation of carbohydrate-active enzymes include the expanded conserved peptide signatures for the AA9, AA10 and AA11 families. As expanded signatures become available for other families, they will be added to Hotpep.

Hotpep could principally be used for annotation of other enzymes than carbohydrate-active enzymes provided that sufficiently well curated sequence data bases are available.

\section{Conclusion}

Hotpep is an easy to use tool that performs automatic annotation of carbohydrate-active enzymes with high success rate. The result of annotation with Hotpep is comparable to state-of-the-art semiautomatic annotation by experts $[1,4]$ and automatic annotation with HMMs [7]. Furthermore, Hotpep also provides a functional prediction of function directly from amino acid sequence.

A downloadable version of Hotpep is available as a stand-alone application that runs on the MS Windows operative system. 


\section{Additional files}

Additional file 1: Conserved Peptide Patterns for all CarbohydrateActive Enzyme Families and CBMs. This file includes all conserved peptide patterns for all PPR groups and functional data for the enzymes in each group. (XLSX $2251 \mathrm{~kb}$ )

Additional file 2: Hotpep annotation of CBMs based on conserved peptides identified by PPR analysis. This file includes the results of Hotpep annotation of CBMs based on conserved peptides identified by PPR analysis with different parameters as indicated. (XLSX $15 \mathrm{~kb}$ )

Additional file 3: Hotpep functional prediction of 8812 experimentally characterized enzymes. This file includes experimental activity data from the CAZy database compared to Hotpep predictions for 8812 carbohydrate-active enzymes. (XLSX 235 kb)

Additional file 4: Comparison of Hotpep annotation from genomes and from predicted proteins. This file includes the results of Hotpep annotation of carbohydrate-active enzymes in seven fungal genomes and in the predicted proteins from the genomes. (XLSX $15 \mathrm{~kb}$ )

\section{Abbreviations}

AA: Auxiliary activities; CBM: Carbohydrate binding module; CE: Carbohydrate esterases; GH: Glycoside hydrolases; GT: Glycosyl transferases; HMM: Hidden Markov Models; Hotpep: Homology to Peptide Pattern; LPMO: Iytic polysaccharide monooxygenase; PL: Polysaccharide lyases; PPR: Peptide Pattern Recognition

\section{Acknowledgements}

We thank Kristian Barrett for fruitful discussions on enzyme annotation and on the performance of Hotpep.

\section{Funding}

This work was supported by project no.: Mar 14319 from Nordic Innovation; SYNFERON - from the Danish Innovation Fund and by The Villum Foundation. The funding bodies did not play any role in the design of the study, in the collection, analysis, and interpretation of data or in writing the manuscript.

\section{Availability of data and materials}

Project name: Hotpep for Carbohydrate-active enzymes

Project home page: https://sourceforge.net/projects/hotpep/

Operating systems: Windows 7 or higher

Programming language: Ruby 2.2.4

License: Creative Commons Attribution-NonCommercial-ShareAlike 4.0 International (CC BY-NC-SA 4.0).

Any restrictions to use by non-academics: Commercial rights reserved.

\section{Authors' contributions}

PKB wrote the software, downloaded the sequences, made the analysis necessary for to develop Hotpep and performed the comparison of annotations. BP tested the Hotpep algorithm and participated in data requisition and analysis. MJL performed the DBCan annotation and result analysis. ASM discussed the final results and interpretation of the data. $L L$ initiated the study and discussed the final results and interpretation of the data. The manuscript was written by the authors from a draft by PKB. All authors read and approved the final manuscript.

\section{Competing interests}

The authors declare that they have no competing interests.
Received: 20 December 2016 Accepted: 5 April 2017 Published online: 12 April 2017

\section{References}

1. Lombard V, Golaconda Ramulu H, Drula E, Coutinho PM, Henrissat B. The carbohydrate-active enzymes database (CAZy) in 2013. Nucleic Acids Res. 2014;42:D490-495.

2. Floudas D, Binder M, Riley R, Barry K, Blanchette RA, Henrissat B, et al. The Paleozoic origin of enzymatic lignin decomposition reconstructed from 31 fungal genomes. Science. 2012;336:1715-9.

3. Grigoriev IV, Martinez DA, Salamov AA. 5 - Fungal Genomic Annotation. In: Dilip K. Arora RMB and GBS, editor. Applied Mycology and Biotechnology [Internet]. Elsevier; 2006 [cited 2016 Dec 1]. p. 123-42. Available from: http:// www.sciencedirect.com/science/article/pii/S1874533406800080

4. Hori $C$, Ishida $T$, Igarashi $K$, Samejima M, Suzuki $H$, Master $E$, et al. Analysis of the Phlebiopsis gigantea Genome, Transcriptome and Secretome Provides Insight into Its Pioneer Colonization Strategies of Wood. PLoS Genet. 2014;10:e1004759.

5. Ekstrom A, Taujale R, McGinn N, Yin Y. PlantCAZyme: a database for plant carbohydrate-active enzymes. Database (Oxford). 2014;2014:bau079.

6. Park BH, Karpinets TV, Syed MH, Leuze MR, Uberbacher EC. CAZymes Analysis Toolkit (CAT): Web service for searching and analyzing carbohydrate-active enzymes in a newly sequenced organism using CAZy database. Glycobiology. 2010;20:1574-84

7. Yin Y, Mao X, Yang J, Chen X, Mao F, Xu Y. dbCAN: a web resource for automated carbohydrate-active enzyme annotation. Nucleic Acids Res. 2012;40:W445-51.

8. Busk PK, Lange L. A Novel Method of Providing a Library of N-Mers or Biopolymers. WO/2012/101151. [Internet]. 2012 [cited 2012 Dec 11]. Available from: http://www.freepatentsonline.com/WO2012101151A1.html

9. Busk PK, Lange L. Function-based classification of carbohydrate-active enzymes by recognition of short, conserved peptide motifs. Appl Environ Microbiol. 2013;79:3380-91.

10. Busk PK, Lange M, Pilgaard B, Lange L. Several genes encoding enzymes with the same activity are necessary for aerobic fungal degradation of cellulose in nature. PLoS One. 2014;9:e114138.

11. Boraston AB, Bolam DN, Gilbert HJ, Davies GJ. Carbohydrate-binding modules: fine-tuning polysaccharide recognition. Biochem J. 2004;382: 769-81.

12. Benson DA, Cavanaugh M, Clark K, Karsch-Mizrachi I, Lipman DJ, Ostell J, et al. GenBank. Nucleic Acids Res. 2013:41:D36-42.

13. Busk PK, Lange L. Classification of fungal and bacterial lytic polysaccharide monooxygenases. BMC Genomics. 2015;16:368.

14. Bech L, Busk PK, Lange L. Cell Wall Degrading Enzymes in Trichoderma asperellum Grown on Wheat Bran. Fungal Genom Biol. 2015;4:116.

15. Brent MR. How does eukaryotic gene prediction work? Nat Biotechnol. 2007;25:883-5

16. Karlsson J, Saloheimo M, Siika-Aho M, Tenkanen M, Penttilä M, Tjerneld F. Homologous expression and characterization of Cel61A (EG IV) of Trichoderma reesei. Eur J Biochem. 2001;268:6498-507.

17. Watanabe T, Kimura K, Sumiya T, Nikaidou N, Suzuki K, Suzuki M, et al. Genetic analysis of the chitinase system of Serratia marcescens 2170. Bacteriol. 1997;179:7111-7.

18. Hemsworth GR, Henrissat B, Davies GJ, Walton PH. Discovery and characterization of a new family of lytic polysaccharide monooxygenases. Nat Chem Biol. 2014;10:122-6

19. Levasseur A, Drula E, Lombard V, Coutinho PM, Henrissat B. Expansion of the enzymatic repertoire of the CAZy database to integrate auxiliary redox enzymes. Biotechnol Biofuels. 2013;6:41.

\section{Consent for publication}

Not applicable.

Ethics approval and consent to participate

Not applicable.

\section{Publisher's Note}

Springer Nature remains neutral with regard to jurisdictional claims in published maps and institutional affiliations. 